\title{
(1) \\ O DESENHO DA FIGURA HUMANA E SEU USO NA AVALIAÇÃO PSICOLÓGICA
}

\author{
The Drawing of the Human Figure and its use in psychological assessment
}

\author{
Roselaine B. Ferreira da Silva ${ }^{[\mathrm{a}]}$, Aline Pasa ${ }^{[\mathrm{b}]}$, Dieime Reis Castoldi ${ }^{[\mathrm{c}]}$, Francieli Spessatto ${ }^{[\mathrm{d}]}$ \\ ${ }^{[a]}$ Doutora em Psicologia (PUCRS), docente-pesquisadora e Coordenadora do Laboratório de Mensuração e Testagem Psicológica \\ no curso de Psicologia da Universidade de Santa Cruz do Sul (UNISC), Santa Cruz do Sul, RS - Brasil, e-mail: mrsilva@unisc.br \\ [b] Acadêmica do Curso de Psicologia da Universidade de Santa Cruz do Sul (UNISC), Santa Cruz do Sul, RS - Brasil. \\ [c] Acadêmica do Curso de Psicologia da Universidade de Santa Cruz do Sul (UNISC), Santa Cruz do Sul, RS - Brasil. \\ ${ }^{[d]}$ Monitora do Laboratório de Mensuração e Testagem Psicológica da Universidade de Santa Cruz do Sul (UNISC), Santa Cruz \\ do Sul, RS - Brasil.
}

\begin{abstract}
Resumo
O presente artigo consiste em apresentar uma breve revisão teórica acerca de um dos instrumentos mais tradicionais na avaliação psicológica: o Desenho da Figura Humana. Aliado a isso, visa a comparar diferentes sistemas de correção e interpretação, destacando semelhanças entre eles. Será enfatizado o instrumento sob a ótica projetiva, apontando a relevância desse na avaliação psicológica. A partir de estudos feitos na disciplina de Técnicas Projetivas II, do curso de Psicologia da Universidade de Santa Cruz do Sul, detectou-se a necessidade de entendimento mais aprofundado acerca desse instrumento. Para tanto, a metodologia empregada para o referente estudo consistiu em busca bibliográfica feita em bancos de dados (PsycInfo, Bireme, Scielo) e literatura da área. A partir dos estudos realizados, destaca-se a importância da técnica projetiva para auxiliar no entendimento da personalidade do sujeito. Foi possível observar que o Desenho da Figura Humana consiste em instrumento muito frequente na avaliação psicológica. Além disso, existem várias propostas de correção dessa técnica, sendo que algumas são validadas e outras caíram em desuso. Existe o interesse em resgatar a proposta de Karen Machover (1967), quanto à solicitação do Desenho da Figura Humana na Chuva. Pela literatura pesquisada, percebe-se que essa técnica consiste em procedimento importante na avaliação psicológica, seja com crianças, adolescentes ou adultos.
\end{abstract}

Palavras-chave: Técnicas projetivas. Desenho da Figura Humana. Validação de instrumentos. 


\begin{abstract}
This article intends to introduce a brief theoretical review about one of the most traditional instruments in psychological assessment: the Drawing of Human Figure. In addition, it aims to compare different systems of correction and interpretation, highlighting similarities between them. The instrument will be emphasized in a projective view, pointing out its relevance in psychological assessment. From studies conducted in the discipline of Projective Techniques II, from Psychology's course at the University of Santa Cruz do Sul, it was detected the need for deeper understanding about this instrument. PsychInfo, Bireme, Scielo databases and literature of the area have been consulted to obtain informations about this instrument. Studies show the importance of projective technique to the understanding of the subject's personality. It was noted that the Drawing of the Human Figure is a very common instrument in psychological assessment. Also there are various proposals to fix this technique; some of which are validated and others fell into disuse. There is interest in rescuing Karen Machover's (1967) proposal on the request of Human Figure Draw in the Rain. According to researched literature, this is an important technical procedure in psychological evaluation, either with children, adolescents or adults.
\end{abstract}

Keywords: Projective techniques. Drawing of the Human figure. Instruments validation.

\section{Avaliação psicológica e o uso de testes}

A avaliação psicológica consiste em processo que permite obter um conhecimento sobre as funções psíquicas do indivíduo, além de investigar sintomas, possibilitando um entendimento mais amplo do caso atendido. Da mesma forma visa à escolha da abordagem terapêutica a ser utilizada com o paciente em questão (Nunes, Silva, Deakin, Dian \& Campezatto, 2006).

Ressalta-se que avaliação e testagem psicológica são conceitos diferenciados. Anastasi e Urbina (2000) definem o teste psicológico como uma medida objetiva e padronizada de uma amostra comportamental e atentam para o fato de que é necessária uma uniformidade de procedimento na aplicação e pontuação do instrumento. $\mathrm{O}$ instrumento sempre será representativo de uma amostra de comportamento que pode ter seus dados analisados estatisticamente, sustentando, dessa forma, suas características psicométricas. Assim, pode ser considerado como um conjunto de itens que medem um determinado fenômeno psicológico (Pasquali, 1999).

Cronbach (1996) afirma ser o termo avaliação mais abrangente que testagem, pois o primeiro se refere mais ao ato de integrar e avaliar informações. Os testes são considerados recursos para esse ato. Para Cunha (2000), a avaliação psicológica consiste em um processo científico, limitado no tempo, no qual podem ser utilizados testes e técnicas para entender problemas psicológicos à luz de pressupostos teóricos.

A princípio, a avaliação psicológica pode se apresentar numa ordem de aparecimento: identificação do fenômeno a ser avaliado (sendo esse o objeto da avaliação), identificação da testagem a ser utilizada e a avaliação propriamente dita, com seus métodos e técnicas inerentes.

Casullo (1999) salienta que o objeto de estudo da avaliação pode ser tanto um sujeito, um grupo, uma instituição ou uma comunidade. Em qualquer caso, deve ser entendido como um sistema complexo, integrado e composto por distintos subsistemas em interação permanente.

Arzeno (1995) esclarece que, muitas vezes, a solicitação de um psicodiagnóstico é demandada pela aplicação de um teste em específico. No entanto, é necessário lembrar que os testes não são um objetivo em si mesmo, mas instrumentos para atingir um fim, e isto é que deve ser esclarecido a quem faz a solicitação.

Outra forma de uso de testagem se dá no espaço terapêutico, com o propósito de estabelecer uma aliança de trabalho em psicoterapia. Nesse sentido, é importante um período inicial de avaliação, sendo esse também constituído pela testagem psicológica. 
Tal como Freud (1995/1911-1913) ressalta em seus escritos, esse processo é importante para que o terapeuta conheça seu paciente, saber qual o motivo que o trouxe à sessão, deixando o paciente falar livremente sobre o que quiser de acordo com o que lhe vem à consciência, sem se preocupar em selecionar esses conteúdos. Esse espaço é significante para a formação do vínculo terapêutico e extremamente importante para um tratamento bem-sucedido. Sendo assim, é nesse período que o profissional pode fazer uso de técnicas para compreender o funcionamento do sujeito que veio procurá-lo, a fim de obter um diagnóstico mais preciso.

Para Werlang (2006), avaliar, no momento prévio ao tratamento, é procedimento importante, pois auxilia o terapeuta a identificar questões não manifestas no paciente. Ao mesmo tempo, auxilia na verificação de problemas mais complexos, possibilitando a priorização de objetivos terapêuticos.

Nesse contexto de avaliação, os testes são utilizados, muitas vezes, com o intuito de proporcionar maior fidedignidade a esse momento. Desta forma, a técnica projetiva do desenho tem sido utilizada em avaliações clínicas. Em alguns momentos, é utilizado para introduzir outras técnicas, como o Rorschach e o Teste de Apercepção Temática (TAT) em adultos, devido à mobilização que esses podem provocar no sujeito.

Para Grassano (1996), a possibilidade de controle intelectual ou de disfarce, seja em âmbito consciente ou inconsciente, é menor nos testes gráficos que nos verbais. O grafismo também pode ser usado como forma de comunicação, principalmente entre as crianças. Tem funcionado como expressão do desenvolvimento geral, pois a partir da maturação gráfica da criança é possível estabelecer as fases do seu desenvolvimento (Campos, 1994; Grassano, 1996; Wechsler, 2003).

Numa perspectiva mais livre, ou seja, na proposta de solicitar um desenho qualquer à criança, em momento avaliativo, Di Leo (1991) compara essa expressão gráfica com o conteúdo dos sonhos. Entende que, tal como o sonho, o desenho também apresenta significados inconscientes. Desta forma, assim como o conteúdo de um sonho diz algo sobre quem sonhou, aquilo que foi desenhado diz algo sobre seu próprio autor.
São inúmeras as modalidades pelas quais pode ser visto o desenho. Ferro (2005) retrata duas delas: o desenho enquanto representação das relações do mundo emocional da criança e o desenho enquanto referência ao funcionamento mental do par terapeuta-paciente.

Fazendo referência à primeira modalidade, o desenho é uma maneira de chegar ao mundo psíquico da criança, além de apresentar elementos que evidenciem a fantasia inconsciente infantil. Já a segunda modalidade prioriza a fantasia transferencial, possibilitando a análise da projeção dos sentimentos da criança sobre o terapeuta.

Ainda Ferro (2005) enfatiza que o desenho necessita ser também verbalizado. Sendo assim, para colocar em palavras aquilo que a criança desenhou se solicita que ela faça associações, e o papel do terapeuta será o de um intérprete.

\section{O Desenho da Figura Humana na avaliação psicológica}

Num primeiro momento, o Desenho da Figura Humana surgiu com uma proposta psicométrica, na tentativa de avaliar características intelectuais. Binet e Simon, por volta de 1905, analisaram o desenho como sendo possível de ser empregado em testes de desenvolvimento mental e aptidões específicas, como também em testes para diagnósticos especiais (Kolck, 1984).

Wechsler (2003) refere que estudos sistematizados com o Desenho da Figura Humana apareceram em torno de 1906, com a investigação de Lamprecht, que comparou os desenhos de crianças de diferentes países tentando encontrar pontos comuns nos seus traçados e conceitos. Igualmente, o pesquisador francês Claparède, em 1907, demonstrou interesse pelos aspectos evolutivos do desenho infantil, na tentativa de averiguar se haveriam relações entre a habilidade para o desenho e a capacidade intelectual da criança demonstrada pelo seu rendimento na escola.

Contudo, foi somente em 1926, com a contribuição de Goodenough, que surgiu um método destinado a avaliar o desenvolvimento intelectual infantil por meio do Desenho da Figura Humana. Assim, desde essa época, o Desenho da Figura Humana tem se revelado como uma das técnicas mais utilizadas para avaliar o desenvolvimento cognitivo, por ser uma medida 
não-verbal conhecida por qualquer criança, de fácil aplicação e baixo custo.

Além do entendimento no nível da cognição, alguns pesquisadores, como Koppitz (1988), Di Leo (1991), Hammer (1991), Cormann (2003) e Machover (1967), entendem que através do desenho é possível realizar a análise da personalidade de um sujeito. Os elementos gráficos falam mais sobre o sujeito do que sobre o desenho propriamente dito. O pressuposto que norteou os estudos desses pesquisadores dizia respeito à ideia de que os desenhos de crianças poderiam ser vistos como indicadores do desenvolvimento psicológico.

Koppitz (1988), com base nos estudos de Goodenough e Machover e trabalhando com crianças de 5 a 12 anos, elaborou uma lista de indicadores emocionais. Consistiu de uma escala própria de índices gráficos que permitem tanto a avaliação do nível de maturação mental, como a detecção e avaliação de distúrbios emocionais (Van Kolck, 1984). A mesma autora apresentou estudos sistematizados sobre os aspectos desenvolvimentais, surgindo um sistema quantitativo-objetivo de avaliação do DFH. ${ }^{1}$ Esse sistema permitiu uma verificação da frequência de itens esperados, comuns, incomuns ou excepcionais para cada faixa etária (Hutz \& Antoniazzi, 1995).

Di Leo (1991) e Hammer (1991) enfatizam que a psicanálise conduziu as pessoas a se conscientizarem de que o inconsciente "fala" em imagens simbólicas. Esses autores identificam que existe uma fase inicial dos desenhos realizados pelo sujeito em que está em jogo não apenas as capacidades intelectuais, como inicialmente eram analisadas, mas também a personalidade dos indivíduos. Segundo a ótica desses autores, pelo desenho é possível investigar os aspectos subjetivos da criança, analisando-se o tamanho, a localização e o conteúdo do que foi desenhado. A pressão do lápis no papel também merece atenção no momento de avaliar o desenho.

Cormann (2003) destaca que o desenho não comporta apenas elementos formais. Ao lado da forma há o conteúdo e nesse se expressam as características de personalidade como um todo.

Machover (1967) publicou os resultados de suas observações clínicas sobre a representação gráfica de figuras humanas desenhadas por crianças e adultos que apresentavam diversos problemas psicológicos, fornecendo, dessa forma, um caráter projetivo ao Desenho da Figura Humana, enquanto método de avaliação da personalidade.

Campos (1994) salienta que o sujeito não desenha apenas o que vê, mas o que sente em adição ao que vê. Esses aspectos podem ser analisados através de alguns itens de correção como o tamanho, localização, pressão do traço e conteúdo do desenho. Wechsler (2003) complementa dizendo que nem sempre a criança desenha o que vê, mas o que sabe sobre si mesma, muitas vezes a partir do que outras pessoas lhe falam. Para Hutz e Bandeira (2000), o desenho também pode ser a representação de outros aspectos do indivíduo, tais como aspirações, preferências, pessoas vinculadas a ele, imagem ideal, padrões de hábitos, atitudes para com o examinador e a situação de testagem. O contexto cultural do sujeito que realizará o teste é outra questão a ser analisada, pois há possibilidade de variações na frequência de alguns itens de correção de acordo com a cultura. Já para Pasian, Okino e Saur (2004), a experiência individual de vida influencia as elaborações projetivas.

De qualquer forma, a premissa da solicitação de um desenho de pessoa, em avaliação psicológica, diz respeito ao fato de que o sujeito projeta uma representação do self: a forma como se vê, assim como acredita que os outros o vêem.

Atualmente, o Desenho da Figura Humana permite avaliar questões tanto cognitivas quanto de personalidade. Resgatar a técnica do Desenho da Figura Humana tem sido o objetivo de alguns pesquisadores. Vidal, Arteche, Hutz e Bandeira (2005) realizaram um estudo sobre o sistema Koppitz para interpretação da Figura Humana. Nesse sentido, verificaram a capacidade discriminativa do instrumento em diferenciar respostas de dois grupos de crianças: com e sem problemas emocionais. Concluíram que apenas os itens "omissão do corpo" $(\mathrm{p}<0,05)$ e "omissão dos braços" $(\mathrm{p}<0,01)$ discriminaram os grupos, sendo mais frequentes na amostra clínica. O restante dos itens não se tornou válido para discriminar crianças com problemas emocionais de crianças sem esses problemas.

Já Bueno, Moura, L'abbate e Pereda (2005) identificaram a relação entre o Desenho da

DFH: Desenho da Figura Humana. No decorrer do texto pode aparecer essa abreviação. 
Figura Humana, traços de personalidade e inteligência. Eles constataram que alguns itens de correção do DFH têm associação com traços de personalidade. Já Bueno, Machado, Schmiedecke e Hamparian (2005) estudaram a relação entre o desenho da casa e traços de personalidade, e demonstraram associação significativa entre esses itens. Assim como o estudo de Castro, Bueno, Dinali, Kebleris e Martinez (2005) associou o desenho da árvore com traços de personalidade, igualmente verificando associação significativa.

Wechsler et al. (2005) analisaram a incidência de indicadores emocionais no Desenho da Figura Humana. Concluíram que existem aspectos desenvolvimentais que influenciam características nos desenhos das crianças e que esses tendem a ser ressaltados, de maneira diferente, por meninos e meninas.

Dessa forma, percebem-se vários estudos, na atualidade, acerca da Figura Humana, enquanto técnica projetiva. Tal fato reforça a ideia de que os pesquisadores estão preocupados com a cientificidade do instrumento. Além disso, diversos encontros científicos voltados à discussão da avaliação psicológica se constituem, cada vez mais, num momento de reflexão e questionamentos sobre a legitimidade das técnicas projetivas enquanto instrumentos efetivamente confiáveis para a avaliação psicológica.

Todavia, os testes gráficos de desenhos necessitam de estudos padronizados para poderem ser autorizados para uso em avaliação psicológica. Nesse sentido, atualmente apenas o HTP (Buck, 2003), o DFH (Sisto, 2005) e o DFH-III (Wechsler, 2003) possuem evidências de validade que demonstram sua eficácia no momento de avaliar uma criança. Dessa forma, cada um deles será retratado a seguir.

\section{Interpretações para o Desenho da Figura Humana}

\section{DFH - III (Desenho da Figura Humana para análise do desenvolvi- mento cognitivo infantil)}

Essa testagem é específica para aplicação em crianças abrangendo as idades de 5 a 12 anos. Está validado, conforme os parâmetros da Resolução
002/2003 do CFP (Conselho Federal de Psicologia), sendo muito utilizado para avaliar o desenvolvimento cognitivo. Sua interpretação se dá em âmbito quantitativo, de acordo com a idade da criança.

A primeira proposta do DFH foi elaborada no ano de 1926, por Goodenough, como análise do desenvolvimento cognitivo. A partir da reformulação da proposta de Goodenough, Wechsler (2003) validou o teste no Brasil.

A aplicação do instrumento é simples, consistindo em solicitar à criança que faça um desenho de um homem e, em seguida, de uma mulher, não necessariamente nessa ordem. A correção é baseada em 30 itens evolutivos que são pontuados como ausentes ou presentes. Atinge-se um escore global a partir da soma dos itens classificados como esperados, comuns, incomuns, e excepcionais, de acordo com a idade da criança (Hutz \& Bandeira, 2000).

Estudo de Silva et al. (2005) destaca que o DFH-III não apresenta influência de questões culturais. Mesmo em contextos diferenciados, como zona urbana e zona rural, as crianças apresentam noção corporal semelhante, além de não evidenciar diferenças significativas na forma de representar as partes do desenho. Mendoza, Abad e Lelé (2005) analisaram os itens de correção que compõem o DFH-III. Verificaram que o teste possui razoável unidimensionalidade, o seja, os itens de correção avaliam o constructo cognitivo.

\section{HTP (Home-Tree-Person)}

Autorizado para uso no Brasil, através da Resolução do CFP, n. 002/2003, esse instrumento foi criado por John Buck em 1949. Anterior a isso, Machover (1967) já tinha realizado alguns estudos com o desenho da pessoa. O objetivo de tais pesquisas era identificar aspectos da personalidade do sujeito que realiza o desenho.

Desta forma, a folha em branco tem a finalidade de representar "o palco" em que serão expressos os estados emocionais da criança, demonstrando o estado emocional no momento da testagem, além de ser possível identificar conflitos mais estruturais de sua personalidade.

$\mathrm{Na}$ técnica do desenho da HTP (CasaÁrvore-Pessoa) solicita-se que o sujeito desenhe, em páginas separadas, o desenho de uma casa, árvore e pessoa. Quanto ao desenho da casa, 
quanto mais estruturada é a sua representação, pode-se dizer que mais adequadas são as condições de funcionamento do ego (Cunha, 2000). São analisados elementos como parede, telhado, chaminé, portas e janelas, sendo que esses últimos estão relacionados ao relacionamento interpessoal.

Quanto ao desenho da árvore, pode-se dizer que são analisados elementos como copa e tronco, bem como a posição do desenho na folha: se está desenhado mais para a direita ou para a esquerda, por exemplo. Esses elementos estão relacionados com o equilíbrio emocional e bom relacionamento com ambos os sexos, assim como a forma de se relacionar com o ambiente (Cunha, 2000).

Já o Desenho da Figura Humana está relacionado aos diferentes aspectos do self. Para alguns pesquisadores, dentre eles Hammer (1991), o desenho da pessoa revela a percepção mais próxima da consciência que o indivíduo tem de si mesmo, ou de um self ideal e de sua relação com o ambiente. Por essa razão, é importante analisar não apenas o desenho, mas também o inquérito solicitado posteriormente ao desenho feito. Essa fase consiste em alguns questionamentos que Buck (2003) sugere, no sentido de aumentar a projeção dos elementos representativos do sujeito em questão.

$\mathrm{Na}$ proposta de correção elaborada por esse autor, são avaliados elementos como proporção do desenho em relação à folha, perspectiva e detalhes. De forma geral, esses podem informar como está o funcionamento de um indivíduo no contexto de seu nível de funcionamento esperado. $\mathrm{O}$ uso apropriado dos detalhes fornece um índice das capacidades do indivíduo para reconhecer elementos do dia a dia. A proporção reflete a capacidade de solucionar problemas básicos e concretos. Já a perspectiva indica como o indivíduo age frente a relacionamentos mais abstratos.

Buck (2003) também propõe a fase do inquérito, sendo esse um subsidio que o avaliador tem para melhor analisar o desenho. Em um primeiro momento a criança faz o desenho e, após o desenho feito, ela conta o que desenhou. Esse é o momento do inquérito, contando com o auxílio de algumas perguntas específicas sobre o desenho. É uma fase essencial para dar à criança a oportunidade de definir, descrever e interpretar cada desenho. No momento em que ela verbaliza o que desenhou, expressa seu pensamento, sentimento e memória, o que auxilia na compreensão de seu mundo interno.
Freitas e Noronha (2005), em seu estudo, destacam que o HTP é um dos instrumentos mais utilizados em processo psicodiagnóstico em uma clínica-escola, sendo usado em diferentes faixas etárias. Dessa forma, se evidencia sua utilização na avaliação clínica.

\section{Desenho da Figura Humana - Escala Sisto}

A Escala Sisto (2005) é indicada para avaliar a inteligência de crianças pequenas, ou com diminuição da capacidade auditiva ou deficiências neurológicas, não-alfabetizadas ou que não falam a língua do examinador. A construção dessa escala teve como base os estudos de Goodenough. É solicitado o desenho de uma pessoa e são analisados, posteriormente, 30 itens de correção, baseados nos critérios de Koppitz.

Esse estudo contribuiu para demonstrar dados estatísticos de validade e precisão, mostrando as diferenças entre os sexos, análise de consistência interna e normas interpretativas. A amostra consistiu em 2.750 crianças, com idade entre 5 a 10 anos, dentre meninos e meninas, distribuídas da pré-escola até a quarta série de escolas públicas e particulares. A aplicação é igualmente simples, sendo solicitado à criança que realize o desenho de uma pessoa. $\mathrm{O}$ autor divide a correção em duas partes: itens imprescindíveis e itens para pontuação.

O trabalho de Sisto (2005) trouxe uma grande contribuição para a área de Avaliação Psicológica, por ser um teste que apresenta estudos confiáveis, com várias evidências de validade.

\section{Desenho da Pessoa na Chuva}

A técnica do Desenho da Pessoa na Chuva não está autorizada para uso no Brasil, pois carece de estudos mais atualizados. Sabe-se que o método projetivo do Desenho da Figura Humana foi proposto por Karen Machover, como forma de investigar a personalidade e características psicológicas (Peres, 2002) e, a partir dessa, surgiram reformulações do teste. É um teste de simples administração, sendo um meio direto para as projeções do sujeito.

Para Machover (1967), o examinador deverá permanecer atento a todos os comentários do examinando durante a aplicação do teste. A solicitação 
é que se desenhe uma pessoa e, depois, deve-se dar outra folha em branco ao sujeito e solicitar que ele desenhe uma pessoa do sexo oposto. Além desses comentários, pode-se aplicar um inquérito após a realização dos desenhos, sendo que há um modelo específico para crianças e outro para adultos.

O Desenho da Pessoa na Chuva, também proposto por Machover (1967), baseia-se no elemento chuva, que tem o caráter simbólico de representar as tensões pelas quais o indivíduo passa. A partir disso, seu objetivo é avaliar como esse enfrenta as situações de estresse.

Como forma de avaliar os desenhos, Machover (1967) destacava aspectos como localização na página, tamanho, qualidade do grafismo, estereótipos (tais como super-heróis) e imagem corporal, que abarca tamanho da cabeça, tronco, mãos, entre outras partes do corpo. Ela afirma que quando um sujeito realiza o desenho está se referindo às imagens internalizadas que tem de si e dos outros. O desenho, assim, representa a expressão do eu no ambiente.

Atualmente existem tentativas de revalidar essa técnica. Um estudo importante foi realizado através de uma pesquisa na cidade de São Paulo (Vagostello, Esteves \& Tardivo, 2007), sendo publicado nos Anais do III Congresso Brasileiro de Avaliação Psicológica. Essa pesquisa foi realizada com crianças vitimizadas e a proposta era verificar como a técnica do Desenho da Pessoa na Chuva poderia contribuir na avaliação psicológica de crianças que sofrem algum tipo de violência. O teste foi aplicado em dois grupos: um composto por 42 crianças que não apresentavam queixas de agressão e 40 que realmente haviam sofrido algum tipo de violência. Os desenhos foram classificados de acordo com a presença ou ausência de oito características encontradas em outro estudo realizado na Argentina: dimensão pequena da figura humana, ausência de pés, ausência de mãos, ausência de detalhes, formato da chuva (chuva como lágrimas, chuva setorizada e raios) e guarda-chuva. Dessas oito características analisadas, três foram sensíveis à detecção de real vitimização: ausência de detalhes, ausência de guarda-chuva e chuva setorizada.

Dessa forma, verifica-se que o Desenho da Pessoa na Chuva é recurso útil para avaliar a dimensão do conflito sentido pelo sujeito, bem como a fragilidade de seu ego frente a situações estressantes. Ao mesmo tempo, pode auxiliar no estabelecimento de vínculos de confiança entre o psicólogo e a criança vitimizada, sendo um meio para que ela expresse seu sofrimento. Detecta-se, assim, o caráter projetivo do teste.

\section{CONCLUSÕES}

A partir das fontes utilizadas no decorrer deste artigo, foi possível observar que o Desenho da Figura Humana é instrumento muito frequente na avaliação psicológica. Além disso, existem várias propostas de correção dessa técnica, sendo que algumas são validadas, e outras caíram em desuso. Como exemplo desse último caso, podemos citar a proposta do Desenho da Pessoa na Chuva de Karen Machover (1967).

No Desenho da Figura Humana podem ser apontados alguns aspectos favoráveis à sua aplicação:

- A linguagem gráfica é a que está mais próxima do inconsciente e do ego corporal;

- Ao contrário da linguagem verbal, mesmo tendo dificuldade em se expressar pela fala, o indivíduo pode realizar o teste;

- Seu conteúdo sofre menor influência do consciente, sendo possível melhor expressão do inconsciente, pois os desenhos, na maioria dos casos, tratam de uma linguagem simbólica;

- Sua administração é simples e econômica;

- No caso de pacientes com dificuldades em se expressar verbalmente, é uma excelente ferramenta para facilitar a comunicação.

Além desses itens, Hutz e Bandeira (2000) também colocam que, além de ser uma técnica de baixo custo, pois se usa apenas lápis e papel, tratase de uma tarefa de fácil execução e muito bem aceita por crianças. Di Leo (1991) ainda destaca que essa é uma das formas de estabelecer um rapport rápido e de forma simples e agradável; fazendo com que a criança sinta-se mais à vontade. $\mathrm{O}$ autor lembra também que nos casos em que os pais têm acesso a esses desenhos, surpreendem-se ao ver como seus filhos os percebem, e como vêem a si mesmos dentro da família. 
Portanto, é a partir de seu desenho que o sujeito expressa a ideia que tem de si mesmo, e qual sua percepção a respeito das outras pessoas que convivem com ele. Para Cunha (2000), quando alguém desenha uma figura humana projeta no papel a forma como se percebe.

Considerando as características encontradas sobre o teste de Machover (1967), deve-se mencionar a importância de novos estudos na área, pois essa técnica envolve muitos elementos simbólicos que estão diretamente relacionados à análise da personalidade do sujeito. Contudo, fazem-se necessários estudos de normatização brasileira, com o intuito de termos normas específicas de correção.

Outro ponto importante diz respeito ao Desenho da Pessoa na Chuva, sendo técnica projetiva que pode trazer elementos significativos frente à forma das pessoas utilizarem seus recursos egoicos nas situações diárias e conflitantes. Tal técnica pode auxiliar o psicólogo em seu trabalho, em especial na avaliação psicológica. Isso porque, dependendo do contexto a ser utilizado (na clínica ou na seleção de pessoal, por exemplo), pode representar um bom subsídio para detecção de problemas ou conflitos na esfera emocional.

Além dessas questões, é possível verificar que essa técnica projetiva necessita de mais estudos que comprovem sua validade para diferentes fins.

\section{CONSIDERAÇÕES FINAIS}

A partir deste estudo, importante destacar a relevância da aplicação de instrumentos psicológicos em avaliação psicológica. O Desenho da Figura Humana merece destaque, tendo em vista sua praticidade e estudos que comprovam sua capacidade em avaliar aspectos da personalidade e, dependendo do sistema de correção, aspectos cognitivos.

Alves (2004) aponta a importância da objetividade durante a correção de qualquer instrumento projetivo, para que esse não se torne uma projeção do examinador, revelando mais aspectos seus do que do examinado. Dessa forma, entende-se que a técnica projetiva do desenho pode ser suscetível a tais questões. Nesse sentido, a preocupação dos pesquisadores não se estende somente ao caráter de validade dos itens de correção do desenho da pessoa, mas também à pessoa que avalia o instrumento.
A prática clínica em psicologia deu às técnicas projetivas um lugar de destaque e de respeito na área da avaliação psicológica. A partir de então, os instrumentos tornaram-se essenciais recursos de acesso a aspectos intrínsecos do sujeito. As pesquisas na área da avaliação psicológica estão se expandindo, mas ainda fazem-se necessárias pesquisas que aumentem a validade e a confiabilidade nos instrumentos voltados à avaliação e à caracterização de aspectos afetivos, estruturais e projetivos.

O caráter projetivo de um teste proporciona o debate entre a avaliação subjetiva e a objetiva. O fenômeno da projeção permite a inferência de aspectos subjetivos. Assim, aquilo que é projetado não é um elemento por si só, único e objetivo, mas a percepção que cada sujeito possui de um mesmo elemento, pleno de significações e expressões da sua personalidade. Considerando tal questão, numa interpretação subjetiva, é possível que os entendimentos de dois avaliadores não coincidam. Frente a um mesmo instrumento ambos chegam a resultados diferentes.

Esse debate acalora a questão da validade de um instrumento projetivo, disparando opiniões divergentes a respeito do tema. De qualquer forma, o desenho da pessoa e o da pessoa na chuva parecem ter, ao longo do tempo, demonstrado evidências clínicas importantes. Alguns pesquisadores tentam retomar essa técnica, com o intuito de também aumentar as possibilidades de técnicas eficientes no trabalho do profissional psicólogo.

\section{REFERÊNCIAS}

Alves, I. C. B. (2004). Técnicas projetivas: Questões atuais na Psicologia. In Vaz, C. E., \& Graeff R. L. (Org.). Técnicas projetivas: produtividade em pesquisa (p. 361). Anais do $3^{\circ}$ Congresso Nacional da Sociedade Brasileira de Rorschach e Outros Métodos Projetivos. Porto Alegre: SBRO.

Anastasi, A., \& Urbina, S. (2000). Testagem psicológica (7a ed.). Porto Alegre: Artmed.

Arzeno, M. E. G. (1995). Psicodiagnóstico clínico: Novas contribuições. Porto Alegre: Artes Médicas.

Buck, J. N. (2003). H-T-P: Casa-Árvore-Pessoa, técnica projetiva do desenho: Manual e guia de interpretação. São Paulo: Vetor. 
Bueno, J. M. H., Machado, A. C. B., Schmiedecke, C. E. V., Hamparian, C. G., \& Silva, D. F. (2005). Relações entre o desenho da casa, traços de personalidade e inteligência. In Instituto Brasileiro de Avaliação Psicológica - IBAP. Desafios para a formação, prática e pesquisa. Anais do $2^{\circ}$ Congresso Brasileiro de Avaliação Psicológica (CD-R). São Paulo: Casa do Psicólogo.

Bueno, J. M. H., Moura, M. M., Labbate, M. F. S., \& Pereda, N. M. (2005). Relações entre o Desenho da Figura Humana, traços de personalidade e inteligência. In Instituto Brasileiro de Avaliação Psicológica - IBAP. Desafios para a formação, prática e pesquisa. Anais do $2^{\circ}$ Congresso Brasileiro de Avaliação Psicológica (CD-R). São Paulo: Casa do Psicólogo.

Campos, D. M. S. (1994). O teste do desenho como instrumento de diagnóstico da personalidade. Petrópolis, Vozes.

Castro, M. R. M., Bueno, J. M. H., Dinali, E. P. L., Kebleris, F., \& Martinez, M. (2005). Relações entre o desenho da árvore, traços de personalidade e inteligência. In: Instituto Brasileiro de Avaliação Psicológica - IBAP. Desafios para a Formação, Prática e Pesquisa. Anais do $2^{\circ}$ Congresso Brasileiro de Avaliação Psicológica (CD-R). São Paulo: Casa do Psicólogo.

Casullo, M. M. (1999). Evaluación psicológica. In S. M. Wechsler., \& R. S. L. Guzzo (Org.). Avaliação psicológica: Perspectiva internacional (pp. 23-39). São Paulo: Casa do Psicólogo.

Corman, L. (2003). O teste do desenho da família. São Paulo: Casa do Psicólogo.

Cronbach, L. J. (1996). Fundamentos da testagem psicológica. (5a ed.). Porto Alegre: Artes Médicas.

Cunha, J. A. (2000). Psicodiagnóstico - V (5a ed.). Porto Alegre: Artmed.

Di Leo, J. H. (1991). A interpretação do desenho infantil (3a ed.). Porto Alegre: Artes Médicas.

Ferro, A. (2005). A Técnica na Psicanálise Infantil - a criança e o analista: Da relação ao campo emocional. Rio de Janeiro: Imago.

Freud, S. (1913/1995). Sobre o início do tratamento (Edição Standard Brasileira das Obras Completas de Sigmund Freud, Vol. 12). Rio de Janeiro: Imago.
Freitas, F. A., \& Noronha, A. P. P. (2005). Clínica-escola: levantamento de instrumentos utilizados em processo psicodiagnóstico. Psicologia Escolar e Educacional, 9(1), 87-93. São Paulo: ABRAPEE.

Grassano, E. (1996). Indicadores psicopatológicos nas técnicas projetivas. São Paulo: Casa do Psicólogo.

Hammer, E. F. (1991). Aplicação clínica nas técnicas projetivas. São Paulo: Casa do Psicologo.

Hutz, C. S., \& Antoniazzi, A. S. (1995). O desenvolvimento do desenho da figura humana em crianças de 5 a 15 anos de idade: normas para avaliação. Psicologia: Reflexão e Crítica, 8(1), 3-18.

Hutz, C. S., \& Bandeira, D. R. (2000). Desenho da figura humana ( $5 \mathrm{a}$ ed.). In Cunha, J. A. Psicodiagnóstico - V (pp. 507-512). Porto Alegre: Artmed.

Koppitz, E. M. (1988). Avaliação psicológica do desenho da figura humana em crianças escolares. Porto Alegre: Artes Médicas.

Machover, K. (1967). O traçado da figura humana: Um método para o estudo da personalidade. In H. H. A., \& G. L. Anderson. (Org.). Técnicas projetivas do diagnóstico psicológico (p. 345370). São Paulo: Mestre Jou.

Mendoza, C. E. F., Abad, F. J., \& Lelé, A. J. (2005). Análise de itens do Desenho da Figura Humana: Aplicação de TRI. Psicologia: Teoria e pesquisa, 20(2), 243-254.

Nunes, M. L. T., Silva, R. B. F., Deakin, E. K., Dian, S. V., \& Campezatto, P. V. M (2006). Avaliação psicológica e a indicação de psicoterapia psicanalítica para crianças. In Werlang, B. G., \& Oliveira, M. S. Temas em psicologia clínica (pp. 29-35). São Paulo: Casa do Psicólogo.

Pasian, S. R., Okino, E. T. K., \& Saur, A. M. (2004). Padrões normativos do Desenho da Figura $\mathrm{Hu}-$ mana em adultos. In Vaz, C. E., \& Graeff, R. L. (Org.). Técnicas projetivas: produtividade em pesquisa. Anais do $3^{\circ}$ Congresso Nacional da Sociedade Brasileira de Rorschach e outros métodos projetivos (pp. 59-66). Porto Alegre: SBRO.

Pasquali, L. (1999). Histórico dos instrumentos psicológicos. In L. Pasquali (Org.). Instrumentos psicológicos: Manual prático de elaboração (pp.13-25). Brasília, DF: LabPAM/IBAPP. 
Peres, R. S. (2002). O Desenho da Figura Humana de Machover aplicado em andarilhos de estrada. In Psicologia, Teoria e Prática, 4(1), 81-92.

Silva, R. B. F., Foresti, M., Tiecher, A., Albers, K., Hoppe, L., \& Scholante, P. (2005). O teste do Desenho da Figura Humana na avaliação do desenvolvimento cognitivo de crianças de um $\mathrm{Mu}$ nicípio do Sul do Brasil - reflexões de uma pesquisa. Barbarói, 22(23), 231-244, EDUNISC.

Sisto, F. F. (2005). Desenho da figura humana: Escala sisto. São Paulo: Vetor.

Vagostello, L., Pinto Jr, A. A., Esteves, C., \& Tardivo, L. S. L. P. C. (2007). Desenho da pessoa na chuva em crianças vítimas de maus tratos no Brasil. In IBAP (Instituto Brasileiro de Avaliação Psicológica). Desafios para a formação, prática e pesquisa. Anais do $2^{\circ}$ Congresso Brasileiro de Avaliação Psicológica (CD-R). São Paulo: Casa do Psicólogo.

Van Kolck, O. L. (1984). Testes projetivos gráficos no diagnóstico psicológico. São Paulo: EPU.

Vidal, M. E. S., Arteche, A., Hutz, C. S., \& Bandeira, D. R. (2005). Indicadores emocionais do Desenho da Figura Humana: um estudo sobre o sistema Koppitz. In Instituto Brasileiro de Avaliação Psicológica - IBAP. Desafios para a Formação, Prática e Pesquisa. $2^{\circ}$ Congresso Brasileiro de Avaliação Psicológica (CD-R). São Paulo: Casa do Psicólogo.

Werlang, B. S. G., \& Oliveira, M. S. (Org.). (2006). Temas em psicologia clínica. São Paulo: Casa do Psicólogo.

Wechsler, S. M. (2003). DFH-III - O desenho da figura humana: Avaliação do desenvolvimento de crianças brasileiras. (3a ed.). Campinas: Impressão Digital.

Wechsler, S., Cunha, G. A., Betanho, S. T., Oliveira, K., Campos, G. R., \& Siqueira, N. F. (2005). Incidência de indicadores emocionais nos desenhos de crianças paulistas. In Instituto Brasileiro de Avaliação Psicológica - IBAP. Desafios para a Formação, Prática e Pesquisa. Anais do $2^{\circ}$ Congresso Brasileiro de Avaliação Psicológica (CD-R). São Paulo: Casa do Psicólogo. 